\section{Erythroleukämoide Reaktion}

H. Baum

Institut für Laboratoriumsmedizin, Mikrobiologie und Blutdepot, Regionale Kliniken Holding RKH GmbH, Ludwigsburg, Deutschland

Englischer Begriff erythroleukemoid reaction

Definition Auftreten kernhaltiger Vorstufen der Erythrozyten sowie unreifer Formen der Granulozytopoese im peripheren Blut im Verlauf von nicht hämatologischen Erkrankungen.

Beschreibung Die erythroleukämoide Reaktion beschreibt das Auftreten von kernhaltigen Vorstufen der Erythropoese, aber auch Vorläuferzellen der Granulopoese im peripheren Blut. Es handelt sich dabei nicht um eine hämatologische Systemerkrankung, sondern das Auftreten erythrozytärer Vorstufen ist sekundärer Natur. Sie ist im Regelfalle ein Ausdruck einer extramedullären Blutbildung. Dabei kommen ursächlich in erster Linie Knochenmetastasen solider Tumoren infrage, die dann sekundär zu einer extramedullären Blutbildung führen.

\section{Literatur}

Begemann H, Begemann M (1997) Praktische Hämatologie, 10., unveränderte Aufl. Georg Thieme Verlag, Stuttgart, S 141 